\title{
Simulation of swelling pressure evolution during infiltration in a bentonite block-pellet laboratory scale test
}

\author{
Ayman A. Abed ${ }^{\text {i) }}$ and Wojciech T. Sołowski ${ }^{\text {ii) }}$
}

\begin{abstract}
i) Postdoctoral Researcher, Department of Civil Engineering, Aalto University, P.O.Box 12100, FI-00076 Aalto, Finland.
\end{abstract} ii) Assistant Professor, Department of Civil Engineering, Aalto University, P.O.Box 12100, FI-00076 Aalto, Finland.

\begin{abstract}
This paper presents numerical simulation of an infiltration test performed on MX-80 block-pellet laboratory sample. The simulation is performed with Thebes Code, a fully coupled THMC finite element program. The focus of the simulation is to recover the observed swelling pressure and dry density of the initially inhomogeneous system during the hydration process and at the final fully saturated state. The numerical results show that the simulation is capable to capture the qualitative response reported in the experimental results. However, the quantitative predictions still need improvements. That could be achieved by better simulation of the three-dimensional geometry of the pellets and further incorporation of the microstructural effects in the employed hydro-mechanical constitutive models.
\end{abstract}

Keywords: nuclear waste, bentonite, coupling, finite element method.

\section{INTRODUCTION}

The final disposal of the spent nuclear fuel poses a serious challenge. In the last decades, many concepts have been proposed for the design of the final disposal sites (Nagasaki and Nakayama, 2015). Among them SKB (Swedish Nuclear Fuel and Waste Management Co) developed the so-called KBS-3V repository design concept, in which the canisters filled with spent fuel are planned to be disposed in specially prepared vertical holes deep in a hosting rock. The canister will be positioned vertically in the middle of the hole being surrounded by compacted bentonite blocks forming an isolation barrier. The design assumes a gap left between the host rock wall and the bentonite blocks. This gap will be filled by bentonite pellet fill with initial dry density that is considerably lower compared to the dry density of the surrounding blocks, see Fig. 1.

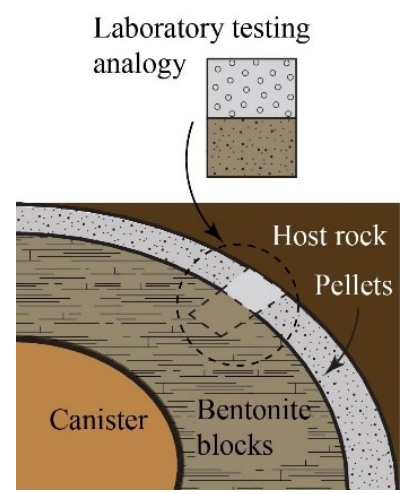

Fig. 1. Isolation arrangements around the spent fuel canister.
During blocks hydration, the swelling pressure will develop and compress the pellets zone, leading to an increase in the dry density. Ideally, at full saturation the final dry density of the blocks and the pellets should become similar. However, laboratory scale experimental results suggest that no full homogenisation will occur. Therefore, the design requires proper recognition of this inhomogeneity so the performance of the proposed sealing barrier stays satisfactory.

This paper presents numerical modelling of an experiment that simulates infiltration into pellet-block sample. The experiment, conducted by Martikainen and Laurila (2018), investigates the interactions in the pellets - bentonite zone (see Fig. 1) and it belongs to a systematic experimental program to study the homogenization process (WP5 of Beacon EU project).

\section{EXPERIMENTAL PROCEDURE AND RSULTS}

Fig. 2 gives the details of the employed testing apparatus and sample in the Martikainen and Laurila (2018) experiment. The numerically replicated experiment, with specification $B-P-1$, used sample of $100.0 \mathrm{~mm}$ height and $70.0 \mathrm{~mm}$ diameter.

The tested sample consists of two zones: 1) compacted bentonite block zone with thickness of $52.0 \mathrm{~mm}$ and 2) pellet fill zone with thickness of $50.0 \mathrm{~mm}$. Fig. 3 shows example of the sample preparation in the actual physical test. It is worthwhile to mention that no powder is used in the pellets zone and the filling is done without any compaction. 


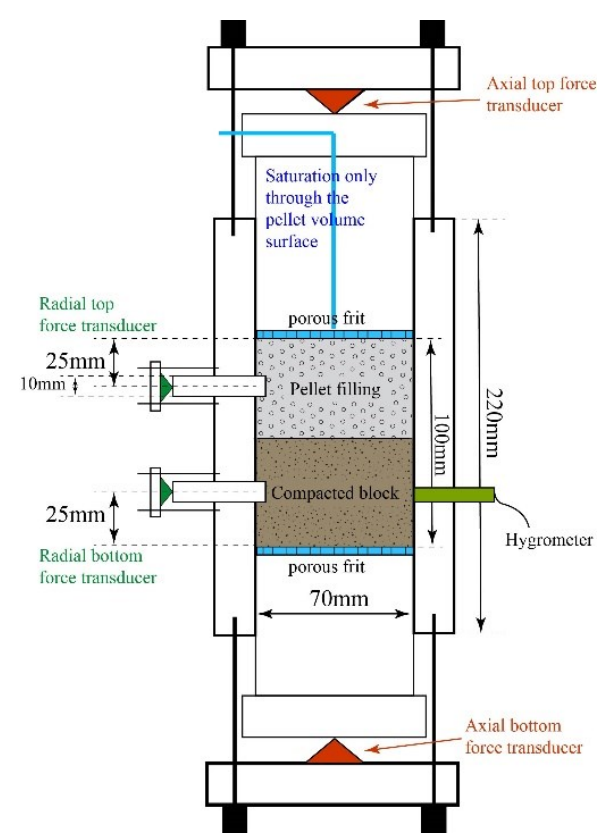

Fig. 2. Testing apparatus with sample geometry (specification BP-1) after Martikainen and Laurila (2018).

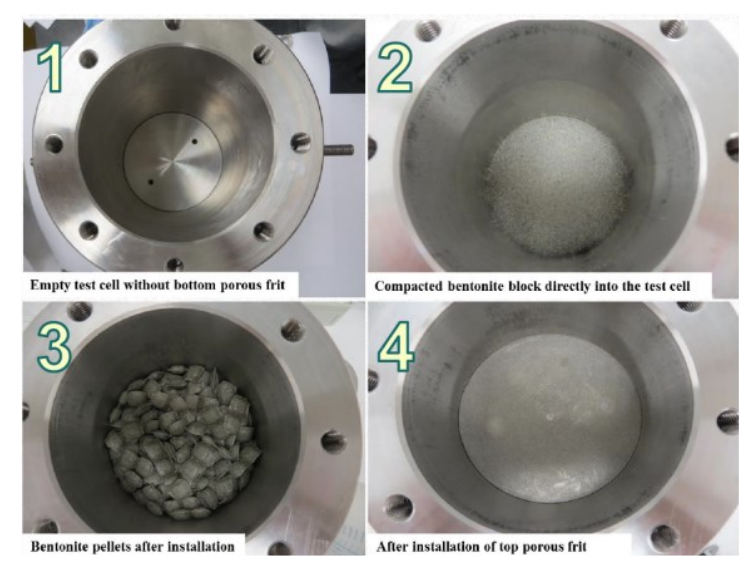

Fig. 3. Phases of sample preparation: 1) empty cell; 2) placement of compacted bentonite block; 3) pellet fill; 4) installation of top porous frit (Martikainen and Laurila, 2018).

Table 1. Relevant properties of the pellets and bentonite block.

\begin{tabular}{lll}
\hline Property & Pellet fill & Block \\
\hline initial dry density $\left[\mathrm{kg} / \mathrm{m}^{3}\right]$ & 919.0 & 1685.0 \\
initial degree of saturation & $22 \%$ & $80 \%$ \\
\hline
\end{tabular}

The hydration of the assembly was performed through the top boundary. The developed axial and horizontal forces were recorded by force transducers positioned at the locations shown in Fig. 2, during the duration of the test, which was 161.0days.

Table 1 lists the relevant properties of the pellets and bentonite block. According to the available testing data, the used pellets identification is Be-Wy-BT0019-Pp-R. This represents small pellets of MX- 80 bentonite with dimensions $12.6 \mathrm{~mm} \times 5.6 \mathrm{~mm}$ (breadth $\times$ thickness) and an initial dry density of $2100 \mathrm{~kg} / \mathrm{m}^{3}$ (Kiviranta et al., 2016; Kiviranta and Kumpulainen, 2011).
Fig. 4 and Fig. 5 show the experimental results in terms of dry density and swelling pressure, respectively. In particular, the swelling pressure measurements in vertical direction seem to be interesting due to the difference between the measured values at the bottom and at the top end of the sample. We associate this imbalance with the friction on the container wall, and thus we introduced such friction in the numerical model. Otherwise, the predicted vertical stress would be the same at both ends due to vertical equilibrium condition.

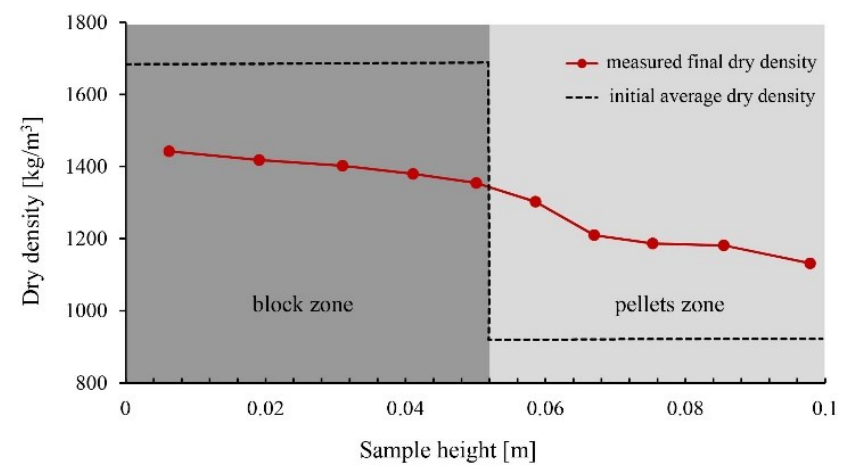

Fig. 4. Initial and final measured dry density after Martikainen and Laurila (2018).

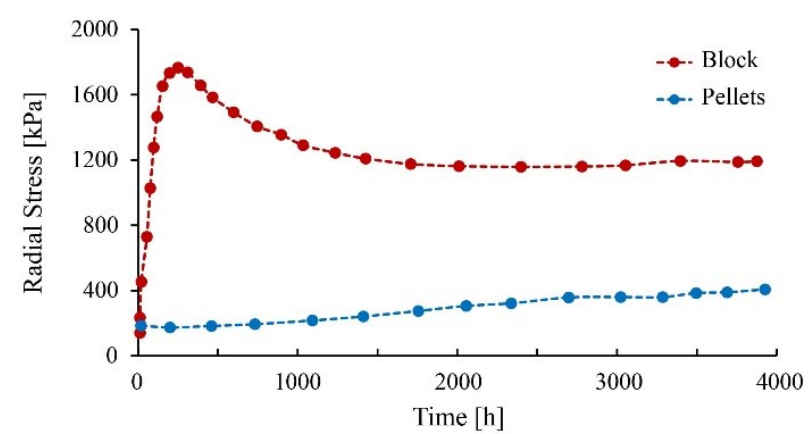

(a)

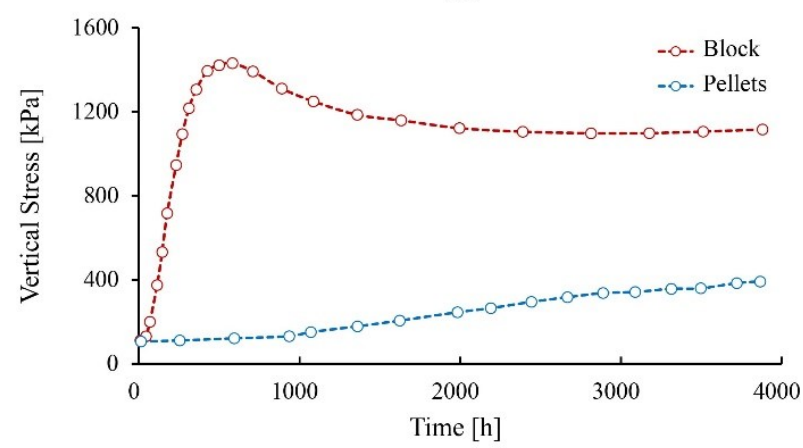

(b)

Fig. 5. Measured swelling pressure: a) in radial direction; b) in vertical direction after Martikainen and Laurila (2018).

\section{MODELLING ASSUMPTIONS}

The test is simulated using Thebes Code, a fully coupled THMC finite element code (Abed and Sołowski, 2017, 2019).

Numerical simulations of similar problems, e.g. Imbert and Villar (2006); Maugis and Imbert ( 2007); 
Gens et al. (2011) and Gens et al. (2013) investigated somehow different material of pellet-powder mixture and treated this mixture as one homogenous material that follows special constitutive model. In contrast to these studies, the presented simulation aims to replicate each individual pellet. However, as it is shown in Fig. 3, the three-dimensional pellets structure complicates the accurate replication of the geometry. The simulation assumes axisymmetry, which leads to an erroneous geometrical representation. Fig. 6 schematically demonstrates that the consequence of the axisymmetry is that the pellets become filled continuous torus.

In order to improve the simulation, the number, the size and the position of the assumed pellet torus are calculated so that the mass of dry bentonite in the pellet zone is conserved. The following section discusses the issue in more details.

\subsection{Mass conservation in axisymmetric conditions}

For the pellet zone the measured initial average dry density is $\rho_{p}^{a v}=919.0 \mathrm{~kg} / \mathrm{m}^{3}$. Considering the dimensions of the simulated cell, the volume occupied by the pellets in the upper part is $V_{p}=\pi(3.5)^{2} \times 5.0=$ $192.422 \mathrm{~cm}^{3}$ and the total bentonite mass is $M_{p}=$ $\rho_{p}^{a v} \times V_{p}=0.919 \times 192.422=176.84 \mathrm{~g}$. Within one radian sector, the amount of bentonite is $M_{p}^{r a d}=$ $28.14 \mathrm{~g} / \mathrm{rad}$. This value represents the reference value that should be conserved during any simulation attempt.

It can be noticed from Fig. 6 that for pellets with constant diameters, the amount of bentonite mass held by each torus sector is proportional to the radial distance. For a single bentonite torus sector represented in Fig. 7, the corresponding volume $V_{p_{i}}$ is:

$$
V_{p_{i}}=2 \pi^{2} R_{i} r_{i}^{2} \frac{\alpha}{360}
$$

where $R_{i}, r_{i}$ and $\alpha$ denote the radial distance to the $i^{\text {th }}$ pellet centre, the pellet radius and the sector angle in degrees, respectively. Consequently, the mass of bentonite contained in each sector is:

$$
M_{p_{i}}=2 \pi^{2} R_{i} r_{i}^{2} \frac{\alpha}{360} \times \rho_{p d}
$$

where $\rho_{p d}=2100 \mathrm{~kg} / \mathrm{m}^{3}$ is the initial pellet dry density. In axisymmetric conditions $\alpha=1 \mathrm{rad}=57.30^{\circ}$ yielding the mass of bentonite in one sector of torus:

$$
M_{p_{i}}=2100 \times \pi R_{i} r_{i}^{2}
$$

If one assumes a constant radius $r$ for the pellets, then the mass will be a single function of the radial pellet position $R_{i}$.

Having the formula (3) and the constraint that the total mass of bentonite in the pellet zone equals $M_{p}^{r a d}=$ $28.14 \mathrm{~g}=0.02814 \mathrm{~kg}$ as discussed earlier, the pellets in the model should always satisfy the condition:

$$
\sum_{i=1}^{m} M_{p_{i}}=0.02814
$$

where $m$ is the total number of assumed pellet torus sectors.

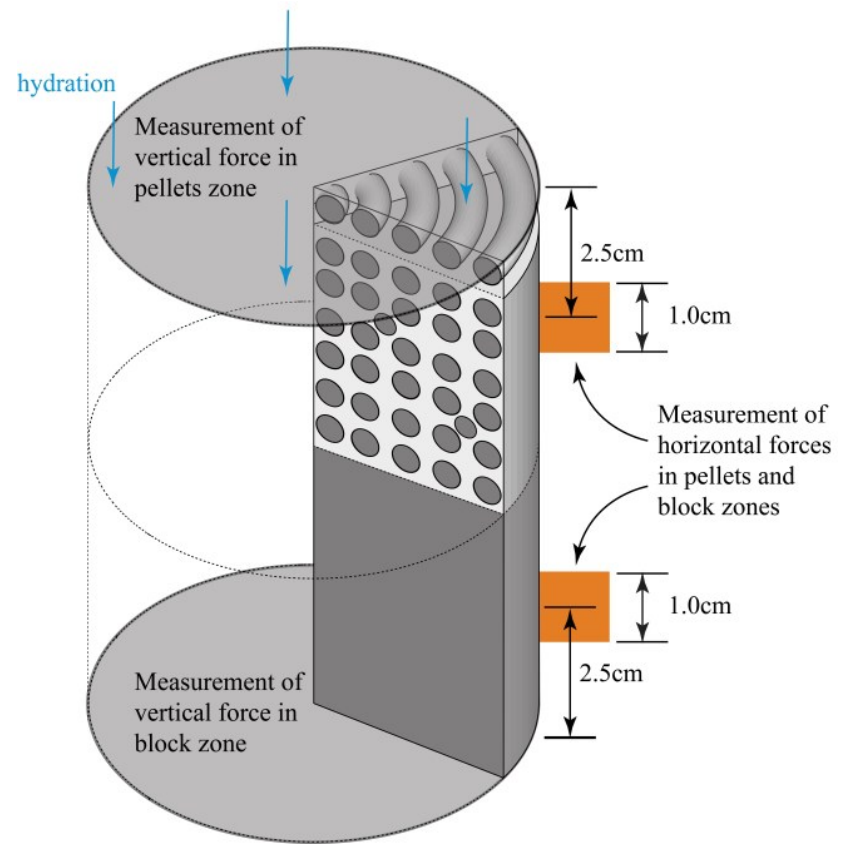

Fig. 6. Resulted geometry in case of axisymmetry assumptions.

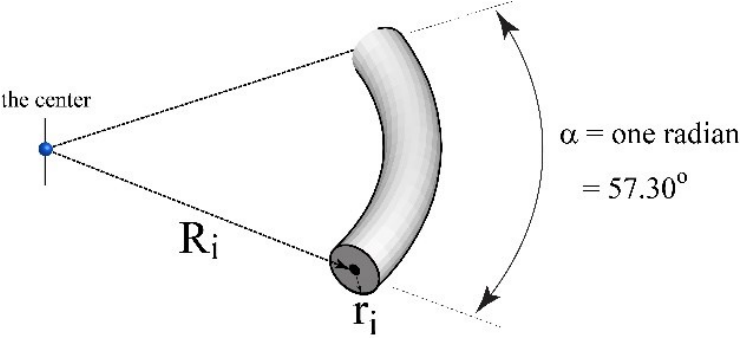

Fig. 7. Single pellet torus sector.

For example, Table 2 demonstrates one possible distribution that conserves mass in the pellets zone assuming a fixed pellet radius $r=3 \mathrm{~mm}=0.003 \mathrm{~m}$, bearing in mind that the radius of the testing cell is $0.035 \mathrm{~m}$. The residual difference of about $0.45 \mathrm{~g}$ is considered to be distributing in the voids among the pellets as dust. The corresponding geometrical distribution is depicted in Fig. 8.

In general, it should be always kept in mind that the circles in this figure and all following comparable figures are in fact representing torus in the full 3D representation.

Table 2. Example of mass-conserving pellets distribution.

\begin{tabular}{lll}
\hline $\mathrm{R}[\mathrm{m}]$ & Number of pellets & Total mass $[\mathrm{kg}]$ \\
\hline 0.005 & 8 & 0.002375 \\
0.0113 & 2 & 0.001342 \\
0.0175 & 8 & 0.008313 \\
0.0238 & 1 & 0.001413 \\
0.03 & 8 & 0.01425 \\
& Sum & 0.027693 \\
& Given & 0.02814 \\
& Difference & 0.000447 \\
\hline
\end{tabular}




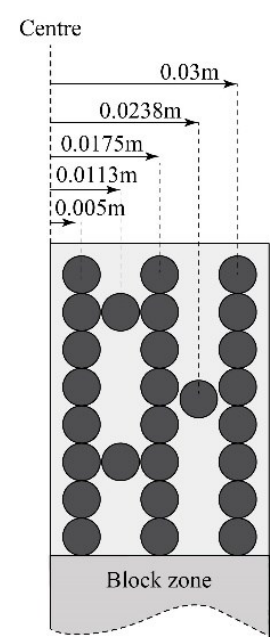

Fig. 8. Possible pellets distribution that conserves the dry mass of bentonite in the pellets zone.

\section{FINITE ELEMENT MODEL}

Fig. 9(a) presents the finite element mesh used in the calculations. It consists of 6000 4-noded quadrilateral elements with 4 Gauss integration points per element. The mesh fineness is chosen after conducting a numerical convergence study. Due to the complex geometry, the assignment of different properties to the different regions (pellets, empty spaces and block zone) are done internally on Gauss point level. The coordinates of the centre of each pellet and the corresponding radius are read from external input file. The position of the current Gauss point is then checked if it is within a possible pellet, in an empty space or in the block zone. Accordingly, suitable hydro-mechanical material properties are assigned. The pellets and the bentonite block are assumed to behave as elastoplastic materials whereas the empty spaces are assumed to be elastic with a very low stiffness. Ideally, the empty spaces should be modelled as gaps in the finite element mesh but that would complicate the simulations and most likely would lead to numerical convergence difficulties at the interfaces of the pellets.

The simulation runs under constant volume conditions, with the mesh constrained against deformation in the normal directions to the boundaries.

Fig. 9(b) illustrates the hydraulic boundary conditions with closed flow boundaries on the sides and open at the bottom.

The hydration is simulated by imposing zero water pressure head at the top boundary. The initial suction is estimated to be equal to $18.0 \mathrm{MPa}$, based on the data provided by Martikainen and Laurila (2018). That suction corresponds to an initial degree of saturation of $80 \%$ in the bentonite block and $22 \%$ in the pellets-voids zone, indicating a coarser material in the latter zone. To capture this difference in initial degree of saturation at similar initial suction, the simulation assumed different soil water characteristic curves for the bentonite block and the pellet-voids zone. The adopted van Genuchten
(1980) parameters for the water retention curves are listed in Table 3. For the bentonite block the parameters were chosen within the range of the measured parameters for MX-80 bentonite (Villar, 2005; KuuselaLahtinen et al., 2016; Abed et al., 2016).

The same saturated hydraulic conductivity $K_{s a t}^{l}=$ $5.0 \mathrm{E}-12 \mathrm{~m} / \mathrm{s}$ is used for the block and the pellets whereas the voids have higher saturated conductivity $K_{s a t}^{l}=1.0 \mathrm{E}-$ $11 \mathrm{~m} / \mathrm{s}$. The voids could have even higher hydraulic conductivity; however, the numerical calibration yielded the values above. This might be partially justified by the slow hydration forced during the physical test (Martikainen and Laurila, 2018).

Fig. 10 shows three different initial configurations for pellets that are designed to conserve mass following the procedure in Section 3.1. The calculations are repeated for these situations and will be referred to them as config(a), config(b) and config(c) according to Fig. 10. The diameters of the pellets are given on the graph. The total number of simulated pellets are 38, 13 and 41 pellets for config(a), config(b) and config(c), respectively.

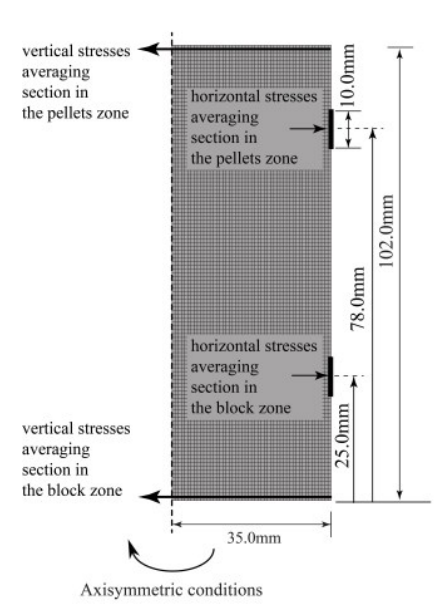

(a)

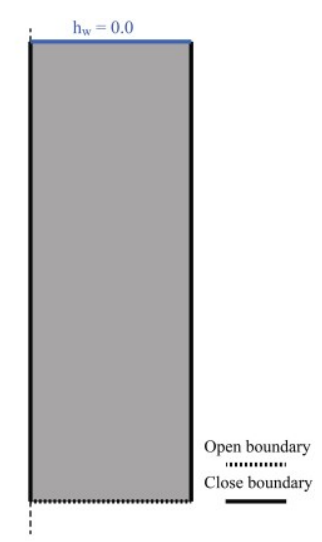

(b)
Fig. 9. Finite element model: a) finite element mesh; b) hydraulic boundary conditions.

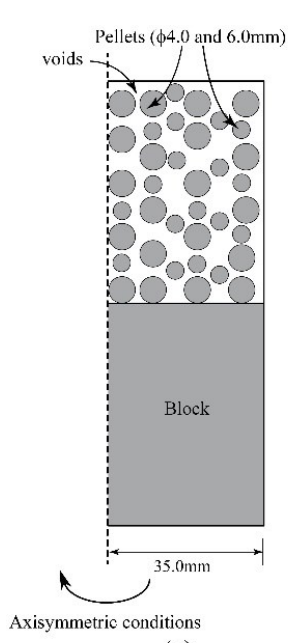

(a)

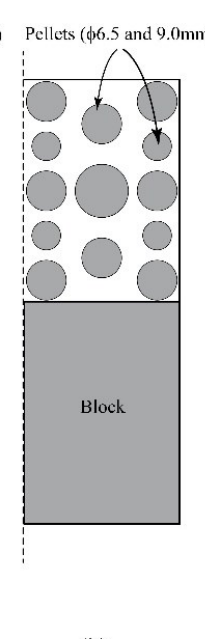

(b)

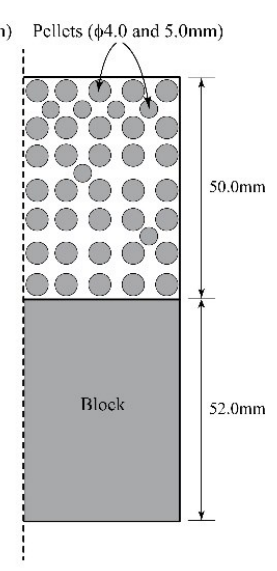

(c)
Fig. 10. Simulated configurations of pellets distribution. 
Table 3 van Genuchten parameters for: a) compacted bentonite blocks and b) voids-pellets zone.

(a) Block zone

\begin{tabular}{ccccc}
\hline$g_{\alpha}\left[\mathrm{m}^{-1}\right]$ & $g_{n}$ & $g_{m}$ & $S_{\text {res }}^{l}$ & $S_{\text {sat }}^{l}$ \\
\hline $3.9 \mathrm{E}-04$ & 1.65 & -0.5 & 0.0 & 1.0 \\
\hline
\end{tabular}

(b) Pellets-voids zone

\begin{tabular}{ccccc}
\hline$g_{\alpha}\left[\mathrm{m}^{-1}\right]$ & $g_{n}$ & $g_{m}$ & $S_{r e s}^{l}$ & $S_{\text {sat }}^{l}$ \\
\hline $5.0 \mathrm{E}-03$ & 1.45 & -0.5 & 0.0 & 1.0 \\
\hline
\end{tabular}

The bentonite block and pellets are simulated using the extended elastoplastic Barcelona Basic Model (BBM) (Gens et al., 2009). That extension of BBM in the elastic region accounts for the expansive nature of these materials. Due to limited space, the features and details of this model will not be given here. The interested reader can find full explanation of this standard model elsewhere, for instance in Alonso et al. (1990); Abed (2008); Gens et al. (2009); Sołowski and Gallipoli (2010) and Abed and Sołowski (2017) among many others. On top of that, the Appendix provides summery of the main equations involved in the extended version of BBM.

The simulation assumes that the empty spaces (gaps) behave as a purely elastic material with low stiffness and considerably high loading-unloading elastic index $\kappa=$ 0.5 . The gaps are insensitive to any suction changes.

Table 4. BBM parameters for block and pellets simulation.

(a) Common parameters for block and pellets

\begin{tabular}{ccccccc}
\hline$v$ & $M$ & $k$ & $\lambda$ & $\beta\left[\mathrm{kPa}^{-1}\right]$ & $r$ & $p^{c}[\mathrm{kPa}]$ \\
\hline 0.3 & 1.07 & 0.001 & 0.15 & $2.0 \mathrm{E}-5$ & 0.8 & 100.0 \\
\hline
\end{tabular}

(b) Specific parameters for each component

(i) Block

\begin{tabular}{cccccccc}
\hline$p_{r e f}^{c}[\mathrm{kPa}]$ & $\kappa_{o}$ & $\kappa_{s o}$ & $\alpha_{k}$ & $\alpha_{k s 1}$ & $\alpha_{k s 2}$ & $e_{o}$ & $p_{o}^{*}[\mathrm{kPa}]$ \\
\hline 10.0 & 0.05 & 0.19 & $-3.0 \mathrm{E}-6$ & -0.145 & 0.0 & 0.573 & 2400.0 \\
\hline
\end{tabular}

(ii) Pellets

\begin{tabular}{cccccccc}
\hline$p_{r e f}^{c}[\mathrm{kPa}]$ & $\kappa_{o}$ & $\kappa_{s o}$ & $\alpha_{k}$ & $\alpha_{k s 1}$ & $\alpha_{k s 2}$ & $e_{o}$ & $p_{o}^{*}[\mathrm{kPa}]$ \\
\hline 2.0 & 0.01 & 0.01 & $-2.0 \mathrm{E}-6$ & -0.145 & 0.0 & 0.262 & 200.0 \\
\hline
\end{tabular}

The block and pellets are assigned typical values of mechanical parameters for MX-80 bentonite that are within the range of the measured parameters in literature, e.g. Kristensson and Åkesson (2008); Toprak et al. (2013) and Abed et al. (2016). However, in order to capture the swelling pressure development, the parameters related to the extended elastic part of BBM are calibrated, these include $\kappa_{o}, \kappa_{s o}, \alpha_{k}, \alpha_{k s 1}$ and $p_{\text {ref }}^{c}$. The final adopted parameters for the provided numerical results in this paper are listed in Table 4 . If needed, the reader might review the Appendix for better understanding of the meaning of these parameters.

Finally, the simulation assumes friction, in order to capture the interaction between the sample and the cell wall. The friction acts in the opposite direction to bentonite displacement is assumed to develop along the external boundary in the axisymmetric simulation. The magnitude of this tangential frictional stress $\tau_{\text {Friction }}$ relates to the normal stress at the boundary $\sigma_{n}$ as:

$$
\tau_{\text {Friction }}=\mu \sigma_{\mathrm{n}}
$$

where $\mu$ is material dependent friction coefficient. The calibration resulted in the values of $\mu_{B}=0.15$ and $\mu_{p}=0.45$ for block and pellets zone, respectively.

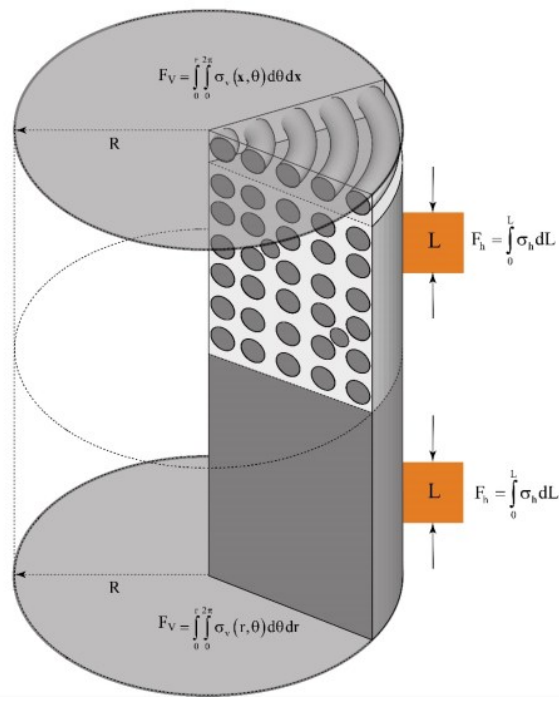

Fig. 11. Stress averaging surfaces.

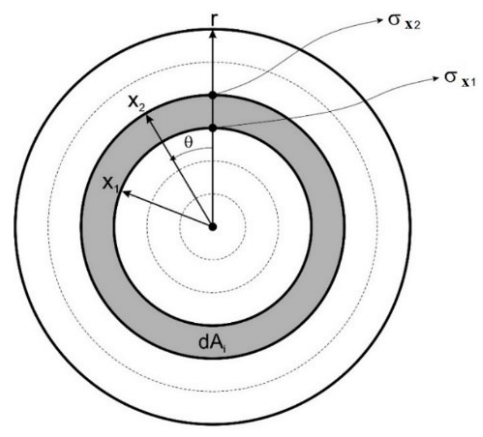

Fig. 12. Top view showing the components used during vertical stress averaging.

\section{STRESS AVERAGING TECHNIQUE}

The discrete nature of pellets in addition to the stiffness differences between the pellets and the surrounding empty spaces result in an inhomogeneous distribution of the vertical stresses at the top boundary. Similarly, the horizontal stresses along the vertical boundary are varying. That means that the monitoring of stresses during numerical simulation is location dependent. Therefore, to achieve a better estimation, the 
stresses are averaged over the approximated measurement area in the experiment. Notably, even though the stress inhomogeneity is more pronounced in the pellets zone, there is still stress inhomogeneity in the block zone due to the friction effect. Therefore, the averaging technique, which is explained in the next paragraph, is used for all the presented results.

Fig. 11 shows the main formulae used for stress integration over the relevant surfaces. The integration of the calculated stresses gives the exerted force on the transducer at that location. Finally, the division of the resulted force over the surface served by the transducer gives the average stress that is compared to the measurements.

\subsection{Averaging of vertical stress}

The exerted vertical force $F_{V}$ relates to the vertical stress $\sigma_{V}$ in the axisymmetric conditions through the integration:

$$
F_{V}=\int_{0}^{R} \int_{0}^{2 \pi} \sigma_{V}(x, \theta) d \theta d x
$$

where $x$ and $\theta$ are the radial and angular position, see Fig. 12. To integrate over the whole top and bottom cross-section of the sample, $x$ goes from 0 to sample radius $R$ whereas $\theta$ goes from 0 to $2 \pi$.

With reference to Fig. 12, in order to benefit from the integration in formula (6) to handle the finite element results it should be converted to a discretized form as follows:

$$
F_{V}=\sum_{i=1}^{n} \sigma_{V_{a v, i}} d A_{i}
$$

where $n$ is the total number of integration slices (for example, one slice is indicated by grey colour in Fig. 12 with $n=6)$. The symbol $\sigma_{V_{a v, i}}$ denotes the average vertical stress acting on the $i^{\text {th }}$ slice which is estimated here as the average value of vertical stresses at $x_{1}$ and $x_{2}$, the slice inner and outer radial position respectively:

$$
\sigma_{V_{a v, i}}=\frac{\sigma_{V_{x_{1}}}+\sigma_{V x_{2}}}{2}
$$

The incremental area $d A_{i}$ is the difference between the areas $A_{2}$ and $A_{1}$ of the outer and inner circles, respectively:

$$
d A_{i}=A_{2}-A_{1}=\pi\left(x_{2}{ }^{2}-x_{1}{ }^{2}\right)
$$

Finally, the average stress is determined as $\sigma_{V}=F_{V} / A$ where $A$ is the given cross section area of the sample.

\subsection{Averaging of radial stress}

The averaged horizontal stresses are relatively easier to determine as they are considered to be simple average of the developed radial stresses over the required area, see Fig. 11.

The considered averaging sections in this study correspond to the position and size of force transducers as indicated in Fig. 2, Fig. 9(a) and Fig. 11.

A macro is written in VisIt visualization software
(VisIt Team, 2003) to export, for each time step, the required calculated stresses along the four sections clarified in Fig. 9(a).

Another special macro is written in MS Excel, which reads the result files exported from VisIt, performs the integration and produces the required averaging plot.

\section{NUMERICAL VERSUS EXPERIMENTAL RESULTS}

\subsection{Swelling pressure}

Fig. 13 and Fig. 14 present the averaged numerical results and the measured data in the case of radial and vertical swelling pressure, respectively. It is clear that the qualitative response of the heterogeneous material is captured acceptably.

In particular, the numerical analyses reproduced the softening part in the vertical swelling pressure with well quantitative agreement, at least for config(a) and $\operatorname{config}(c)$. Unfortunately, this observation does not apply for the radial swelling pressure. In the latter case, the maximum radial swelling pressure is captured but without significant softening after the peak value. It is worth noting that, using exactly the same modelling parameters, the three pellets configurations yielded these comparable results.

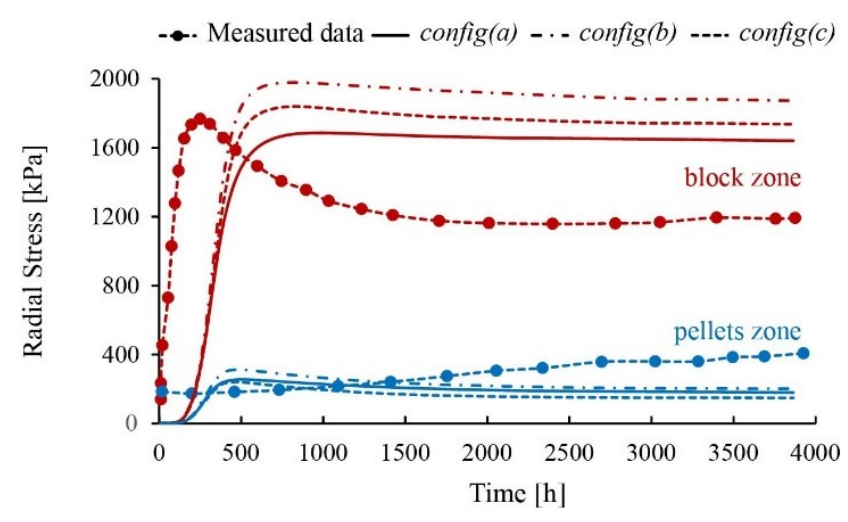

Fig. 13. Measured radial swelling pressure versus finite element averaged results for three different pellets configurations.

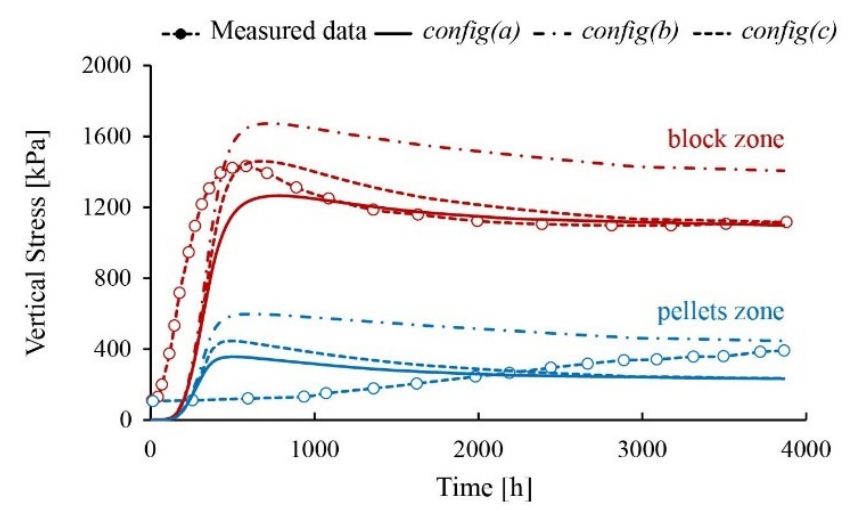

Fig. 14. Measured vertical swelling pressure versus finite element averaged results for three different pellets configurations. 


\subsection{Dry density}

The numerical results of dry density in the pellets zone show obvious scattering. That is expected due to the difference between the initial dry density of the pellets and the empty spaces around them. An example of this scatter is shown in Fig. 15 where the blue squares represent the direct numerical results. Two methods are used to process the scatter: 1) adopt simple average of all numerical values in the pellets zone which yielded a value of $1062 \mathrm{~kg} / \mathrm{m}^{3}$ for config $(a)$. The straight line in brown in Fig. 15 represents this value. 2) Another way is to average locally around the measured values. The second method resulted in the red line in Fig. 15, which looks more realistic following the trend of the measured data. The local averaging is used also to average the calculated final dry density in the case of $\operatorname{config}(b)$ and config(c) as shown in Fig. 16.

Again, the qualitative variation in dry density is as expected with decreasing value in the block zone and increasing value in the pellets zone due to swelling pressure. Nonetheless, the predicted values are still considerably deviating from the measured data.

Finally, Fig. 17 shows graphical output for vertical and radial stresses as well as dry density at the end of the simulation in case of config $(a)$. The total water flux is also shown but after 4.0days of test start.

\section{CONCLUSIONS}

The current study follows a new methodology to approach the modelling of the block-pellets system by considering each individual pellet in the simulation. The ability of this method is tested against the experimental data by Martikainen and Laurila (2018).

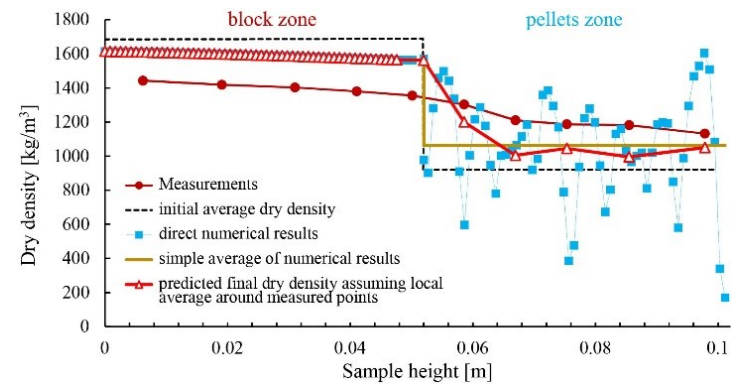

Fig. 15. Resulted final dry density in case of $\operatorname{config}(a)$ versus measured values.

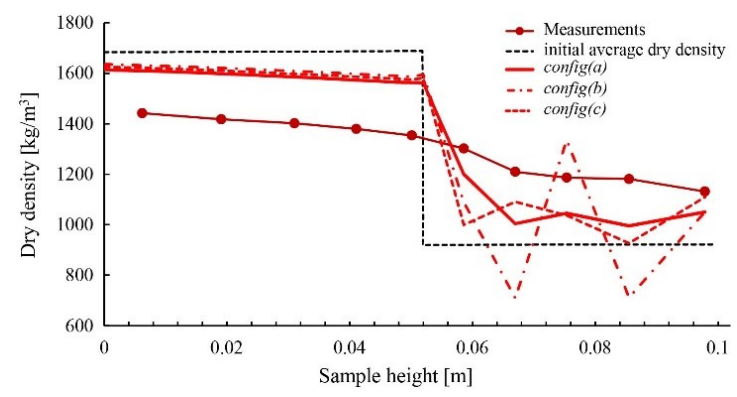

Fig. 16. Measured final dry density versus averaged finite element results for three different pellets configurations.

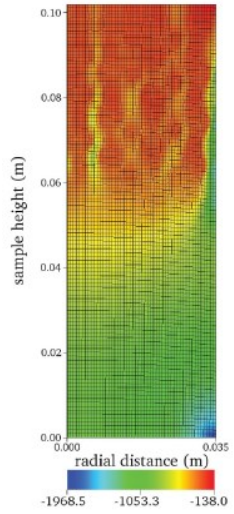

(a)

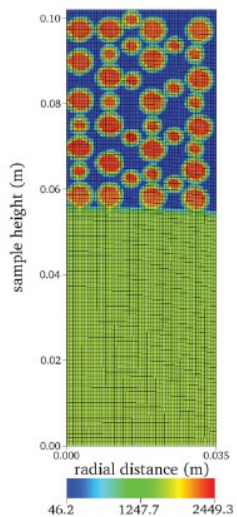

(c)

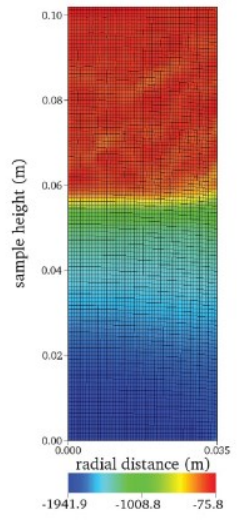

(b)

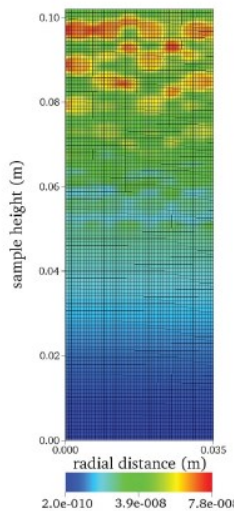

(d)
Fig. 17. Graphical output: a) vertical stress in $[\mathrm{kPa}]$ after 161 days; b) radial stress in $[\mathrm{kPa}$ after 161 days; c) dry density in $\left[\mathrm{kg} / \mathrm{m}^{3}\right]$ after 161 days; d) total water flux in $[\mathrm{m} / \mathrm{s}]$ after 4.0days.

The paper shows numerical simulation performed under axisymmetric conditions. To compensate the error due to this strong assumption, the pellets configuration is chosen so it satisfies the conservation of dry mass of bentonite in the sample. The research also assessed the influence of the initial pellets configuration by simulating three different initial packing, each time using exactly the same constitutive parameters.

To overcome the non-uniform distribution of stresses due to discrete pellets, the stresses are averaged over the sections in focus. The simulation also introduced friction between the bentonite and cell walls.

In general, the followed methodology is able to reproduce qualitatively the observed experimental trend of the swelling pressure, vertical stress and the dry density variation during hydration and at full saturation. Different pellets configurations yielded comparable numerical results with potentially better results with the increasing number of simulated pellets. The differences could be partially explained by the difference in the resulted wall friction as well as the stress averaging technique.

The quantitative predictions are still considerably different from the recorded data. A better simulation would be achieved by considering the full 3D geometry of the problem and enhancing the employed hydro- 
mechanical constitutive models by including microstructure effects. The results reported in this paper are elementary but highlight important points that need to be improve in future research.

\section{ACKNOWLEDGEMENTS}

The authors would like to gratefully acknowledge that the presented research has been funded by KYT2018 Finnish Research Programme on Nuclear Waste Management via THEBES project. Special thanks are due to Dr Lasse Lavikainen from Posiva Oy in Finland for providing the data for the infiltration test.

\section{REFERENCES}

1) Abed, A. (2008): Numerical modeling of expansive soil behavior, Instituts für Geotechnik (IGS).

2) Abed, A. and Sołowski, W. (2017): A study on how to couple thermo-hydro-mechanical behaviour of unsaturated soils: Physical equations, numerical implementation and examples, Computers and Geotechnics, 92, 132-155.

3) Abed, A. and Sołowski, W. (2019): Applications of a New THMC Coupled Code "Thebes", Environmental Geotechnics (in press).

4) Abed, A., Laitinen, M., Lämsä, J., et al. (2016): Hydromechanical modelling of MX-80 bentonite: one dimensional study, Proceeding of E-UNSAT 2016, Paris, France.

5) Alonso, E., Gens, A. and Josa, A. (1990): A constitutive model for partially saturated soils, Géotechnique 40(3), 405430.

6) Beacon EU project (WP5) (2019): Deliverable D5.1.1. 'Specifications of verification/validation test cases-geometry, characteristics of the material, measures', (in press).

7) Gens, A., Sánchez, M., Guimaraes, N., et al. (2009): A fullscale in situ heating test for high-level nuclear waste disposal: observations, analysis and interpretation, Géotechnique, 59(4), 377-377.

8) Gens, A., Valleján, B., Zandarín, M., et al. (2013): Homogenization in clay barriers and seals: Two case studies, Journal of Rock Mechanics and Geotechnical Engineering, 5(3), 191-199.

9) Gens, A., Vállejan, B., Sánchez, M., et al. (2011): Hydromechanical behaviour of a heterogeneous compacted soil:experimental observations and modelling, Géotechnique 61(5), 367-386.

10) Imbert, C. and Villar, M. (2006): Hydro-mechanical response of a bentonite pellets/powder mixture upon infiltration, Applied Clay Science, 32(3-4), 197-209.

11) Kiviranta, L. and Kumpulainen, S. (2011): Quality control and characterization of bentonite materials, Posiva Oy, Finland.

12) Kiviranta, L., Kumpulainen, S., Pintado, X., et al. (2016): Characterization of bentonite and clay materials, Posiva Oy, Finland.

13) Kristensson, O. and Åkesson, M. (2008): Mechanical modeling of MX-80-Quick tools for BBM parameter analysis, Physics and Chemistry of the Earth, 33, 508-515.

14) Kuusela-Lahtinen, A., Sinnathamby, G., Mendez, J., et al. (2016): Estimation of water retention behaviour of MX-80 bentonite partially saturated with saline solution, Proceeding of E-UNSAT 2016, Paris, France.

15) Martikainen, J. and Laurila, T. (2018): Laboratory Tests to Evaluate Bentonite Homogenization, Internal Posiva memorandum. Helsinki: Saanio \& Riekkola Oy.

16) Maugis, P. and Imbert, C. (2007): Confined wetting of FoCa clay powder/pellet mixtures: Experimentation and numerical modeling. Physics and Chemistry of the Earth, 32(8-14), 795-808.

17) Nagasaki, S. and Nakayama, S. (2015): Radioactive Waste Engineering and Management. Springer.

18) Sołowski, W. and Gallipoli, D. (2010): Explicit stress integration with error control for the Barcelona Basic Model: Part I: Algorithms formulations, Computers and Geotechnics, 37(1): 59-67.

19) VisIt Team (2003): VISIT: Software the Delivers Parallel Interactive Visualization, Lawrence Livermore National Laboratory, http://www. llnl. gov/visit.

20) Toprak, E., Mokni, N., Olivella, S., et al. (2013): Thermohydro-mechanical modelling of buffer, Posiva Oy, Finland.

21) Van Genuchten, M. (1980): A closed-form equation for predicting the hydraulic conductivity of unsaturated soils, Soil science society of America journal, 44(5), 892-898.

22) Villar, M. (2005): MX-80 bentonite, thermo-hydromechanical characterisation, Ciemat, Madrid, Spain.

\section{APPENDIX}

In order to account for the swelling nature of some soils, the elastic stiffness parameters in the Barcelona Basic Model are made isotropic net pressure $p$ and suction $s$ dependent as follows:

$$
\kappa=\kappa_{o}\left(1+\alpha_{\kappa} s\right) ; \quad \kappa_{s}=\kappa_{s o}\left(1+\alpha_{\kappa s 1} \ln \left(\frac{p}{p_{r e f}^{c}}\right)\right) \mathrm{e}^{\alpha_{\kappa s 2} s}
$$

where $\kappa_{o}$ and $\kappa_{s o}$ are reference values of $\kappa$ and $\kappa_{S}$, the swelling index with respect to net stress and suction, respectively. Extra material parameters $\alpha_{\kappa}, \alpha_{\kappa s 1}, \alpha_{\kappa s 2}$ and $p_{r e f}^{c}$ are needed in this case.

To fully describe the elastic behaviour, the Poisson's ratio $v$ and the initial void ratio $e_{o}$ should also be provided to the model. For plasticity, the yield surface is given as:

$$
F=q^{2}-M^{2}\left(p+p_{s}\right)\left(p_{o}-p\right)=0
$$

where $M$ is the slope of critical state line and $q$ is the deviatoric stress.

BBM assumes that the shear strength is affected by suction and temperature increment $\Delta T$ with $p_{s}=k s e^{-\rho^{T} \Delta T}$ where $\rho^{T}$ and $k$ are material constants.

The soil preconsolidation pressure $p_{o}$ is also considered to be suction and temperature dependent:

and

$$
\begin{gathered}
p_{o}=p^{c}\left(\frac{p_{o T}^{*}}{p^{c}}\right)^{\frac{\lambda-\kappa}{\lambda_{s}-\kappa}} \text { with } \lambda_{s}=\lambda\left[(1-r) \mathrm{e}^{-\beta s}+r\right] \\
p_{o T}^{*}=p_{o}^{*}\left(1-\gamma_{T} \log \left(\frac{T}{T_{o}}\right)\right)
\end{gathered}
$$

where $\lambda$ is the slope of normal consolidation line. The preconsolidation pressure at full saturation and at the reference temperature $T_{o}=20{ }^{\circ} \mathrm{C}$ is indicated by $p_{o}^{*}$. The parameters $p^{c}$, $\beta, r, \quad \gamma_{T}$ are material constants that have to be experimentally determined. 